FERMILAB-Conf-00/009-E

CDF/PUB/BOTTOM/CDFR/5204

November 21, 2018

\title{
SPECTROSCOPY AND LIFETIME $\mathrm{OF}$ BOTTOM AND CHARM HADRONS
}

\author{
FUMIHIKO UKEGAWA \\ Institute of Physics, University of Tsukuba \\ Tsukuba, Ibaraki 305-8571, Japan \\ The CDF Collaboration
}

Invited talk at the

3rd International Conference on $B$ Physics and $C P$ Violation

Taipei, Taiwan

December $3-7,1999$ 
This is a blank page. 


\title{
SPECTROSCOPY AND LIFETIMES OF BOTTOM AND CHARM HADRONS
}

\author{
Fumihiko Ukegawa \\ (CDF Collaboration) \\ Institute of Physics, University of Tsukuba \\ Tsukuba, Ibaraki 305-8571 Japan \\ E-mail: ukegawa@physics.px.tsukuba.ac.jp
}

We review recent experimental results on spectroscopy and lifetimes of bottom and charm hadrons.

\section{Introduction}

There are several motivations for studying masses and lifetimes of the hadrons containing a heavy quark, either the bottom or the charm quark. First, the mass and the lifetime are fundamental properties of an elementary particle. Second, the spectroscopy of hadrons gives insight into the QCD potential between quarks. In particular, a symmetry exists 1 for heavy hadrons when the heavy quark mass is taken to be infinite, providing a powerful tool to predict and understand properties of those heavy hadrons. Third, studies of the lifetimes of heavy hadrons probe their decay mechanisms. A measurement of the lifetime, or the total decay width, is necessary when we extract magnitudes of elements of the Kobayashi-Maskawa matrix 2 . Again, in the limit of an infinite heavy quark mass things become simple and the decay of a heavy hadron should be the decay of the heavy quark $Q$. This leads to a prediction that all hadrons containing the heavy quark $Q$ should have the same lifetime, that of the quark $Q$. This is far from reality in the case of charm hadrons, where the $D^{+}$meson lifetime is about 2.5 times longer than the $D^{0}$ meson lifetime. Perhaps the charm quark is not heavy enough. The simple quark decay picture should be a better approximation for the bottom hadrons because of the larger $b$ quark mass.

On the experimental side, the measurements and knowledge of the heavy hadrons (in particular bottom hadrons) have significantly improved over the last decade, thanks to high statistics data accumulated by various experiments. We shall review recent developments in these studies in the remainder of this manuscript. 

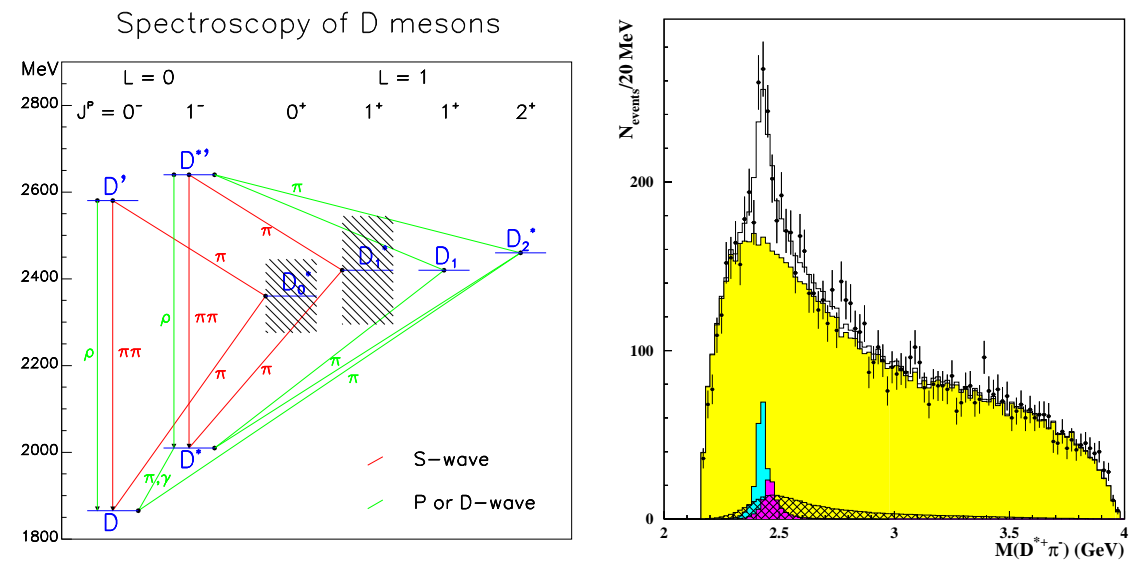

Figure 1: Left: Spectrum of charm mesons. Right: $D^{*+} \pi^{-}$mass spectrum by CLEO.

\section{Charm Hadron Spectroscopy}

Let us consider mesons consisting of a charm quark $c$ and a light antiquark $\bar{q}$ (Figure 1 1 (left)). The ground states are the pseudoscalar $(D)$ and the vector $\left(D^{*}\right)$ mesons, which have long been established. They have zero orbital angular momentum $(L=0)$ between the quarks, and the total angular momentum of the mesons are given by addition of two spin $1 / 2$ particles, namely $J=S=0$ or 1 .

If we allow for one unit of orbital angular momentum $(L=1)$ and combine it with the total spin $S$ of the $c \bar{q}$ system, there will be four states, $J^{P}=1^{+}$, $0^{+}, 1^{+}$and $2^{+}$. These states are sometimes called the $D^{* *}$ mesons. They are expected to decay predominantly to $D^{(*)} \pi$ pairs via the strong interaction. Angular momentum and parity conservation restrict the decay of the $0^{+}$meson to the $D \pi$ final state. Similarly, the $1^{+}$meson decays only to $D^{*} \pi$ pairs. The $2^{+}$meson can decay either to $D^{*} \pi$ or $D \pi$.

In the limit of an infinite heavy quark mass, $m_{Q}=\infty$, the light degree of freedom is decoupled from the heavy quark, and the total angular momentum of the light antiquark $j=L+s_{\bar{q}}$ is a good quantum number. In this case the four states above form two doublets, each having $j=1 / 2(J=0,1)$ or $j=3 / 2(J=1,2)$, where two members of each doublet are degenerate in mass, width and other quantum numbers. The decay of the $j=3 / 2$ doublet proceeds through a $D$-wave, while the decay of the $j=1 / 2$ doublet proceeds through an $S$-wave. Therefore, the $j=3 / 2$ doublet is expected to be narrow and the $j=1 / 2$ doublet to be wide. 
The two narrow states, $D_{1}$ and $D_{2}^{*}$ mesons in the PDG notations, have been established 3 . Here we describe the first observation of a wide state by the CLEO experiment 6 . CLEO uses $3.1 \mathrm{fb}^{-1}$ of $e^{+} e^{-}$annihilations at the $\Upsilon(4 S)$ resonance. The $B^{-} \rightarrow D^{*+} \pi^{-} \pi^{-}$decay is used, and the $D^{*+} \pi^{-}$pairs are studied. The $D^{*+}$ meson is reconstructed in the $D^{0} \pi^{+}$decay mode. The usage of this decay chain gives enough constraints that allow the study of the $D^{*+} \pi^{-}$pairs without explicitly reconstructing the $D^{0}$ meson, resulting in large increase in statistics. In particular, the angular correlations that arise from the fact that the $D^{* *}$ meson is fully polarized in the decay are exploited intensively. Figure 1 1 (right) shows the $D^{*+} \pi^{-}$mass spectrum. The background subtracted distributions are also shown, as are the two narrow states, as well as a wide component (hatched histogram). The mass and width of the wide component are measured to be $m=2461_{-34}^{+41} \pm 10 \pm 32 \mathrm{MeV} / c^{2}$ and $\Gamma=$ $290+79 \pm 26 \pm 36 \mathrm{MeV} / c^{2}$. Also the branching fraction is measured to be $\mathcal{B}\left(B^{-} \rightarrow D_{1}^{* 0} \pi^{-}\right) \cdot \mathcal{B}\left(D_{1}^{* 0} \rightarrow D^{*+} \pi^{-}\right)=(10.6 \pm 1.9 \pm 1.7 \pm 2.3) \times 10^{-4}$.

The DELPHI experiment reported 10 evidence for a radial excitation state $D^{* /+}$ using the $D^{*+} \pi^{+} \pi^{-}$final state. However, neither OPAL nor CLEO has been able to confirm it 6 .

CLEO has found 7 a new charmed baryon state that decays through $\Xi_{c}^{*} \pi$ to $\Xi_{c} \pi^{+} \pi^{-}$. Both charged and neutral states are observed as a peak in the mass difference $\Delta m \equiv m\left(\Xi_{c} \pi^{+} \pi^{-}\right)-m\left(\Xi_{c}\right)$, at $348.6 \pm 0.6$ (charged) and $347.2 \pm 0.7 \mathrm{MeV} / c^{2}$ (neutral). An upper limit on the width is placed to be $\Gamma<3.5$ (charged) and $<6.5$ (neutral) $\mathrm{MeV} / c^{2}$. They are interpreted as the $J^{P}=\frac{3}{2}^{-} \Xi_{c 1}$ isospin doublet, namely the $L=1$ orbital excitation of the $\Xi_{c}$ baryon.

\section{Bottom Hadron Spectroscopy}

A lot of progress has been made in the spectroscopy of bottom hadrons in the past few years as well. All ground states have been established, and even the $B_{c}^{-}$meson, a bound state of the two different kinds of heavy quarks, has been observed 6 . The $B^{*}$ mesons have also been established, at a mass about 50 $\mathrm{MeV} / c^{2}$ above $B$. The mass splitting is smaller than in the charm mesons $\left(\sim 150 \mathrm{MeV} / c^{2}\right)$, certainly consistent with expectations from the heavy quark symmetry.

The $B^{* *}$ states have also been observed. Here we describe an L3 analysis 9 as a recent example. The $B$ mesons are reconstructed inclusively using secondary vertices and are combined with pion candidates coming from the primary vertex. Figure 2 (left) shows the $B \pi$ mass distribution, where an en-

hancement is observed around $5.7 \mathrm{GeV} / c^{2}$. A fit for the masses and widths of 

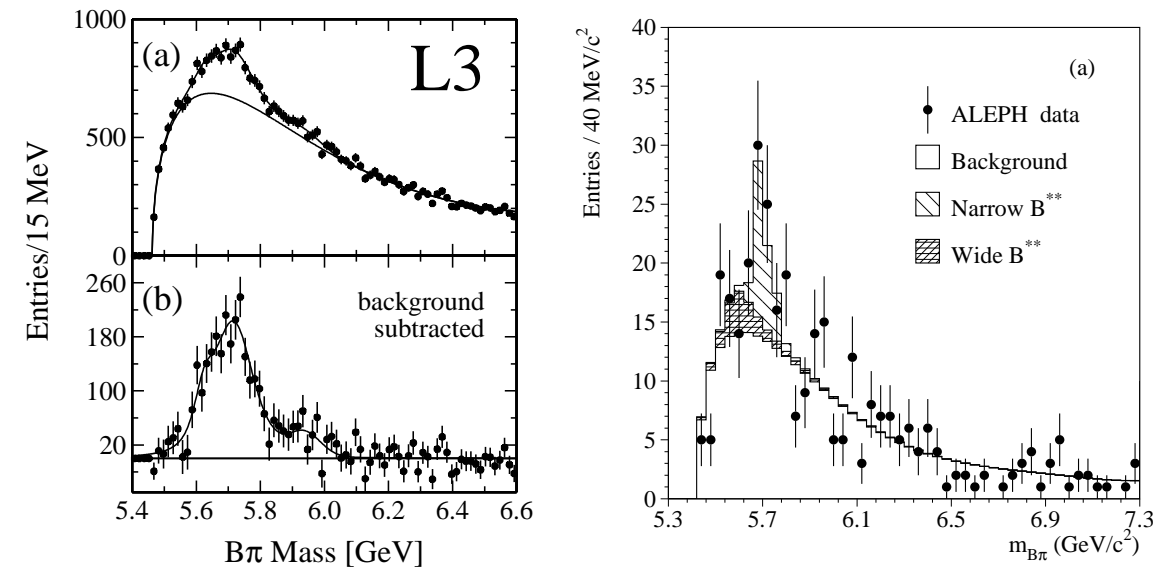

Figure 2: $B^{* *}$ analyses by L3 (Left) and ALEPH (Right). $B \pi$ mass spectra are shown.

the narrow and the wide states is performed, assuming, within each doublet, the mass splitting of $12 \mathrm{MeV} / c^{2}$ and equal widths, and relative production rates according to $2 J+1$ spin counting. This yields $m=5768 \pm 5 \pm 6 \mathrm{MeV} / c^{2}$ and $\Gamma=24 \pm 19 \pm 24 \mathrm{MeV} / c^{2}$ for the $B_{2}^{*}$ state, and $m=5670 \pm 10 \pm 13$ $\mathrm{MeV} / c^{2}$ and $\Gamma=70 \pm 21 \pm 25 \mathrm{MeV} / c^{2}$ for the $B_{1}^{*}$ state. The fraction of the $B^{* *}$ production is extracted to be $f\left(b \rightarrow B^{* *} \rightarrow B^{(*)} \pi\right)=0.32 \pm 0.03 \pm 0.06$. The fit actually includes a fifth component, radial excitation states $B^{\prime}$, to account for an enhancement near $5.9 \mathrm{GeV}$, giving $m=5937 \pm 21 \pm 4 \mathrm{MeV} / c^{2}$, $\sigma=50 \pm 22 \pm 5 \mathrm{MeV} / c^{2}$, and $f\left(b \rightarrow B^{\prime} \rightarrow B^{(*)} \pi\right)=0.034 \pm 0.011 \pm 0.008$.

Although the $B^{* *}$ mesons are collectively observed, their individual states have not been separated. This is because (a) typical $B$ reconstruction relies on inclusive techniques and thus suffers from a relatively poor mass resolution, (b) the photon in the $B^{*} \rightarrow B \gamma$ decay is not usually included and thus $B^{*} \pi$ and $B \pi$ states are not separated, and (c) all $B$ hadron species are mixed together.

OPAL 10 attempted to remedy the point (b) above, with a statistical separation of $B^{*} \pi$ and $B \pi$ states by looking for the photon from the $B^{*}$ decay. Inclusively reconstructed $B$ decay events are separated into $B^{*}$-enriched and $B^{*}$-depleted samples, and the $B^{* *}$ branching fraction of $\mathcal{B}\left(B^{* *} \rightarrow B^{*} \pi\right)=$ $0.85_{-0.27}^{+0.26} \pm 0.12$ is extracted.

ALEPH $⿴$ uses fully reconstructed decays $B \rightarrow \bar{D}^{(*)} n \pi$ and $J / \psi K^{(*)}$, solving difficulties (a) and (c). Signals of about $200 B^{+}$and $140 B^{0}$ are observed. The $B \pi$ mass spectrum is shown in Figure 2(right). The limited statistics, however, prevent the determination of the masses and widths of all 
four states separately. A fit similar to that in the L3 analysis is performed, assuming a mass splitting within a doublet of $12 \mathrm{MeV} / c^{2}$, a mass splitting between the narrow and wide doublets of $100 \mathrm{MeV} / c^{2}$, and widths $\Gamma\left(B_{2}^{*}\right)=25$ $\mathrm{MeV} / c^{2}, \Gamma\left(B_{1}\right)=21 \mathrm{MeV} / c^{2}$ and $\Gamma=150 \mathrm{MeV} / c^{2}$ for wide states, as well as relative production rates according to $2 J+1$ spin counting. The fit yields $m\left(B_{2}^{*}\right)=5739_{-11-4}^{+8} \mathrm{MeV} / c^{2}$ and $f\left(b \rightarrow B^{* *} \rightarrow B^{(*)} \pi\right)=0.31 \pm 0.09_{-0.05}^{+0.06}$. $\mathrm{CDF} 2$ performs a similar analysis using semileptonic decays $\bar{B} \rightarrow \ell^{-} \bar{\nu} D^{(*)} X$.

There is one important application of $B^{* *}$ mesons for other $B$ physics studies, namely flavor tagging. It is an essential ingredient for measurements of $B^{0} \bar{B}^{0}$ oscillations and some $C P$ violation phenomena. It has been customary to infer the flavor of the $B$ hadron of interest by identifying the flavor of the other $B$ hadron in the event, using the fact that $b$ and $\bar{b}$ quarks are produced in pairs. It is also possible to exploit the charge-flavor correlation between the $B$ hadron and the nearby charged pion 13 . A well known example would be the decay $D^{*+} \rightarrow D^{0} \pi^{+}$when you look for $D^{0} \bar{D}^{0}$ mixing. If it is accompanied by $\pi^{+}$, it must have been produced as $D^{0}$, not $\bar{D}^{0}$. The $B^{*}$ mesons cannot produce a pion kinematically. Therefore, $B^{* *}$ mesons are the main resonant state that can produce correlated pions. They can also be produced in the fragmentation (non-resonant) processes. The charge correlations are the same whether it is resonant or non-resonant The method has been applied successfully to $B^{0} \bar{B}^{0}$ oscillation measurements 4 . The ALEPH analysis above also performs tagging studies and finds similar tagging effectiveness. Tag purity could be improved further if the $B \pi$ mass resonant regions are selected.

\section{Charm Hadron Lifetimes}

Recent developments in charm lifetime measurements include (a) a new precision in the $D_{s}^{+}$lifetime (E791, CLEO) and (b) startup of new experiments (FOCUS, SELEX). Here we describe the $D_{s}^{+}$lifetime measurements.

E791 15 uses data from 1991 - 92 with $500 \mathrm{GeV} / c \pi^{-}$beam on foil targets. The $D_{s}^{+}$meson is reconstructed with the $\phi \pi^{+}, \phi \rightarrow K^{+} K^{-}$mode. Figure $3($ left $)$ shows the $\phi \pi^{+}$mass spectrum. A signal of $1662 \pm 56$ is estimated. The peak around $1860 \mathrm{MeV} / c^{2}$ is from the Cabibbo-suppressed $D^{+}$decay. The dashed line represents combinatorial background, where a discontinuity arises because the $D_{s}^{+} \rightarrow \phi \pi^{+}$candidates were required to be inconsistent with the $D^{+} \rightarrow K^{+} \pi^{-} \pi^{+}$hypothesis. Mass and decay time distributions are fit simultaneously and the $D_{s}^{+}$lifetime is extracted to be $\tau\left(D_{s}^{+}\right)=518 \pm 14 \pm 7$ fs. Using the $D^{0}$ lifetime of $415 \pm 4$ fs (PDG 1998), it is found that $\tau\left(D_{s}^{+}\right) / \tau\left(D^{0}\right)=1.25 \pm 0.04$.

CLEO 16 uses $3.7 \mathrm{fb}^{-1}$ of $e^{+} e^{-}$annihilation near the $\Upsilon(4 S)$. The $D_{s}^{+}$, as 

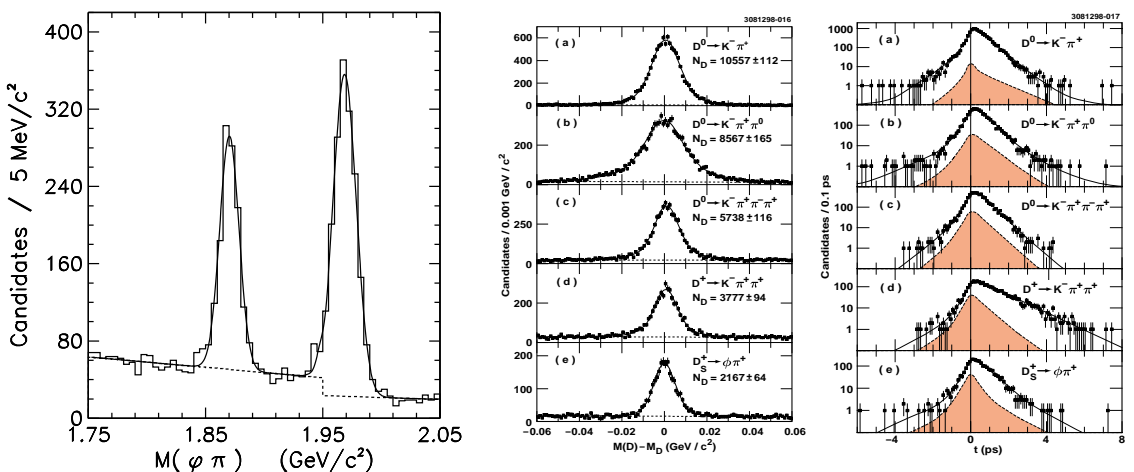

Figure 3: Left: $D_{s}^{+}$lifetime measurements by E791. Right: Charm lifetime measurements by CLEO.

well as $D^{0}$ and $D^{+}$mesons are reconstructed. Figure 3 (right) shows the reconstructed mass (difference from the nominal mass) and decay time distributions. They find $\tau\left(D^{0}\right)=408.5 \pm 4.1_{-3.4}^{+3.5} \mathrm{fs}, \tau\left(D^{+}\right)=1033.6 \pm 22.1_{-12.7}^{+9.9} \mathrm{fs}, \tau\left(D_{s}^{+}\right)=$ $486.3 \pm 15.0_{-5.1}^{+4.9} \mathrm{fs}$, and the lifetime ratio of $\tau\left(D_{s}^{+}\right) / \tau\left(D^{0}\right)=1.19 \pm 0.04$.

Each result establishes for the first time that the $D_{s}^{+}$lifetime is significantly longer than the $D^{0}$ lifetime.

\section{Bottom Hadron Lifetimes}

The bottom hadrons are expected to have smaller lifetime differences among the different hadron species, of order $10 \%$ at most 17 . This poses a challenge to experiments, because it requires great precision to find and establish such small differences. The $B$ hadron lifetime measurements performed thus far can be classified into three broad classes, using (a) inclusive $B$ reconstruction, (b) partial reconstruction (e.g. semileptonic decays) and (c) full reconstruction (e.g. $J / \psi K^{(*)}, D^{(*)} n \pi$ ). Method (a) gives the largest statistics, but samples are less pure in terms of isolating the various $B$ hadron species, and the selection procedure introduces a bias in the proper time distribution of the candidates that needs to be carefully accounted for. Method (b) gives respectable statistics and sample purity. Method (c) gives clean signature, perfect purity, and very precise momentum estimate on an event-by-event basis, but suffers from small branching fractions and thus low statistics. Here we describe two measurements, using method (a) and (b), respectively.

SLD 18 uses inclusively reconstructed $B$ decays among $550 \mathrm{k}$ hadronic $Z^{0}$ decays. The net charge of tracks attached to the secondary vertices is used to 

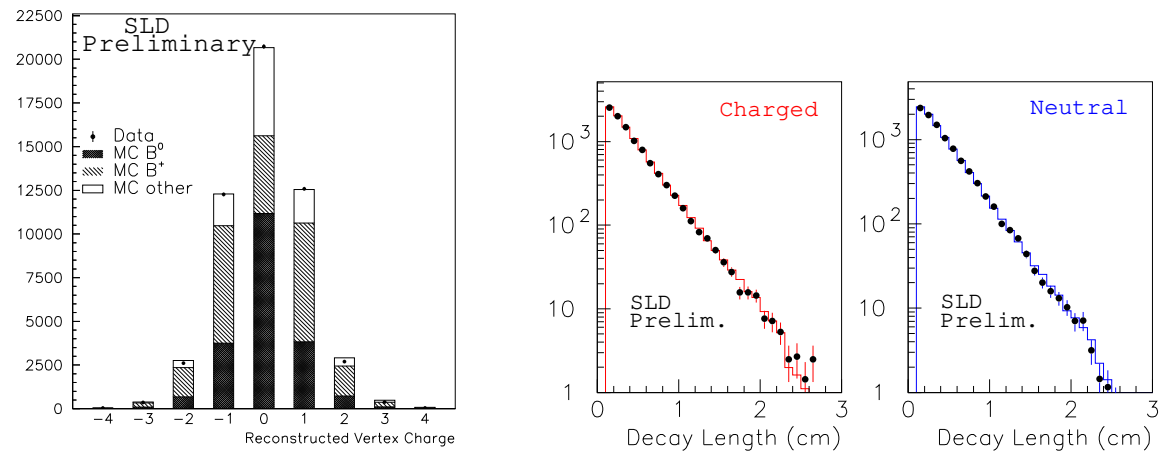

Figure 4: $B^{+}$and $B^{0}$ meson lifetime measurement by SLD. Left: vertex charge distribution. Right: decay length distributions for charged and neutral $B$ hadron samples. The plots show the 1997 - 98 data corresponding to $350 \mathrm{k}$ hadronic $Z^{0}$ events.

estimate and separate the charge of parent $B$ hadrons. The purity is enhanced further with information from the vertex mass, the beam polarization and the opposite hemisphere jet charge. Figure 1 shows the distributions of the vertex charge and the decay lengths for samples enriched in charged and neutral $B$ hadrons. They find $\tau\left(B^{+}\right)=1.623 \pm 0.020 \pm 0.034 \mathrm{ps}, \tau\left(B^{0}\right)=1.565 \pm 0.021 \pm$ $0.043 \mathrm{ps}$, and the lifetime ratio of $\tau\left(B^{+}\right) / \tau\left(B^{0}\right)=1.037_{-0.024}^{+0.025} \pm 0.024$.

ALEPH 19 has re-analyzed $4 \mathrm{M}$ hadronic $Z^{0}$ decays taken in $1991-95$ and measured the $B^{+}$and $B^{0}$ meson lifetimes using semileptonic decays $\bar{B} \rightarrow$ $\ell^{-} \bar{\nu} D^{*+} X$ (mostly $\bar{B}^{0}$ ) and $\bar{B} \rightarrow \ell^{-} \bar{\nu} D^{0} X$ (mostly $B^{-}$). The $D^{*+}$ meson is reconstructed in the $D^{0} \pi^{+}$mode, and the $D^{0}$ meson is reconstructed in $K^{-} \pi^{+}, K^{-} \pi^{+} \pi^{+} \pi^{-}, K^{-} \pi^{+} \pi^{0}$ or $K_{S}^{0} \pi^{+} \pi^{-}$mode. The charm signals are shown in Figure 5 . For the $\ell^{-} D^{0}$ pairs, the $D^{0}$ meson is reconstructed in $K^{-} \pi^{+}, K^{-} \pi^{+} \pi^{0}$ or $K_{S}^{0} \pi^{+} \pi^{-}$mode, and decays coming from the $D^{*+}$ meson are excluded. Similar signals are observed. Distributions of the $B$ meson proper decay time are also shown in Figure 5. The measured lifetimes are $\tau\left(B^{+}\right)=1.646 \pm 0.056_{-0.034}^{+0.036} \mathrm{ps}, \tau\left(B^{0}\right)=1.524 \pm 0.053_{-0.032}^{+0.035} \mathrm{ps}$, and the lifetime ratio is $\tau\left(B^{+}\right) / \tau\left(B^{0}\right)=1.080 \pm 0.062 \pm 0.024$.

These two results are shown as recent representative measurements. There are many other measurements from various experiments, as compiled by the LEP $B$ lifetime working group, and more information can be found in Ref.20. As of October 1999, the world average lifetimes of $B^{+}$and $B^{0}$ mesons are $\tau\left(B^{+}\right)=1.639 \pm 0.025 \mathrm{ps}, \tau\left(B^{0}\right)=1.553 \pm 0.029 \mathrm{ps}$, and $\tau\left(B^{+}\right) / \tau\left(B^{0}\right)=$ $1.066 \pm 0.024$. The value at the time of the Hawaii Conference (March 1997) was $\tau\left(B^{+}\right) / \tau\left(B^{0}\right)=1.06 \pm 0.04$. Namely, the precision is still improving, and 

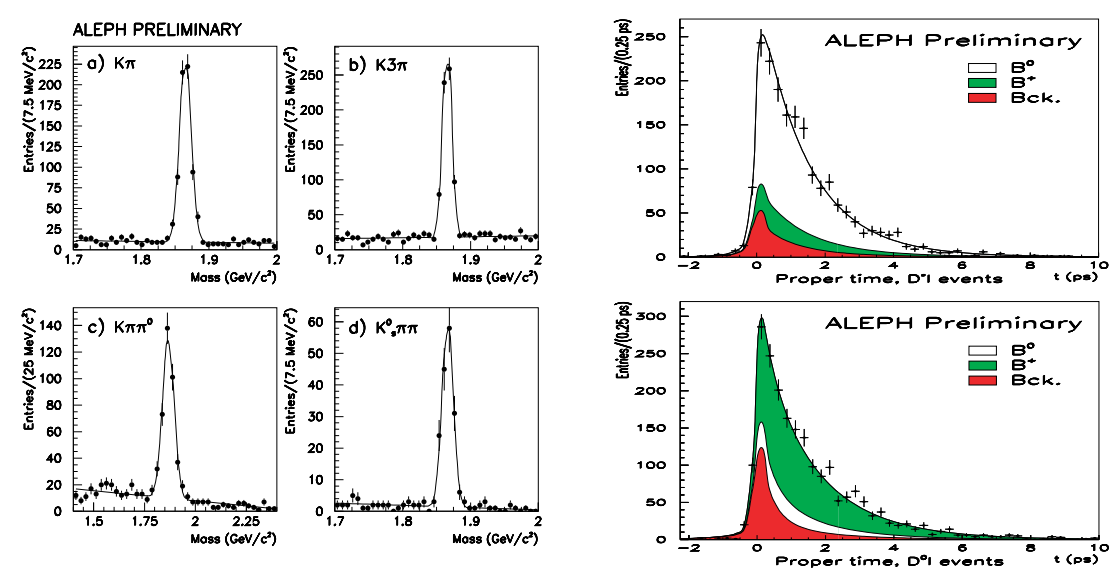

Figure 5: $B^{+}$and $B^{0}$ meson lifetime measurement by ALEPH. Left: $D^{0}$ signal from $D^{*+} \rightarrow D^{0} \pi^{+}$associated with $\ell^{-}$. Right: $B$ meson proper decay time distributions for $\ell^{-} D^{*+}$ and $\ell^{-} D^{0}$ samples.

we may be on the verge of observing a finite lifetime difference.

For the $B_{s}^{0}$ meson, a very small (of order $1 \%$ ) lifetime difference from the $B^{0}$ meson is expected. The current world average value is $\tau\left(B_{s}^{0}\right)=1.460 \pm$ $0.056 \mathrm{ps}$, and $\tau\left(B_{s}^{0}\right) / \tau\left(B^{0}\right)=0.94 \pm 0.07$. More precise measurements are necessary in order to see whether there is a lifetime difference of order $5 \%$, or whether the lifetimes agree at $1 \%$ level.

Another interesting piece of physics is expected in the $B_{s}^{0}$ meson system. The $B_{s}^{0}$ meson should mix with its antiparticle to form the mass eigenstates $B_{s, L}^{0}$ and $B_{s, H}^{0}$, where the subscripts $L$ and $H$ denote light and heavy states. These two mass eigenstates could also have a substantial width difference $\Delta \Gamma$ of order $10 \%$. A sizable width difference is interesting for several reasons: (a) we should see it if it indeed exists, (b) the ratio $\Delta m_{s} / \Delta \Gamma$ is independent of CKM matrix elements and is estimated to be large $(\sim-180)$, providing an indirect information on $\Delta m_{s}$, (c) it may allow $C P$ studies using untagged decays 21, and (d) physics beyond the Standard Model should make $\Delta \Gamma$ smaller.

The width difference $\Delta \Gamma$ manifests itself as a difference in lifetimes when measured with flavor eigenstates (e.g. semileptonic decays) and $C P$ eigenstates. Or, the decay time distributions of flavor eigenstates can be fitted with two (long andshort) components to look for a width difference.

DELPHI 22 uses $3.5 \mathrm{M} Z^{0}$ decays and the semileptonic decay $\bar{B}_{s}^{0} \rightarrow$ $\ell^{-} \bar{\nu} D_{s}^{+} X$. The $D_{s}^{+}$meson is reconstructed in $\phi \pi^{+}, \bar{K}^{* 0} K^{(*)+}, K_{S}^{0} K^{+}, \phi \pi^{+} \pi^{+} \pi^{-}$ or $\phi \pi^{+} \pi^{0}$ mode, as well as the semileptonic mode $\phi \ell^{+} \nu$. A total signal of 290 

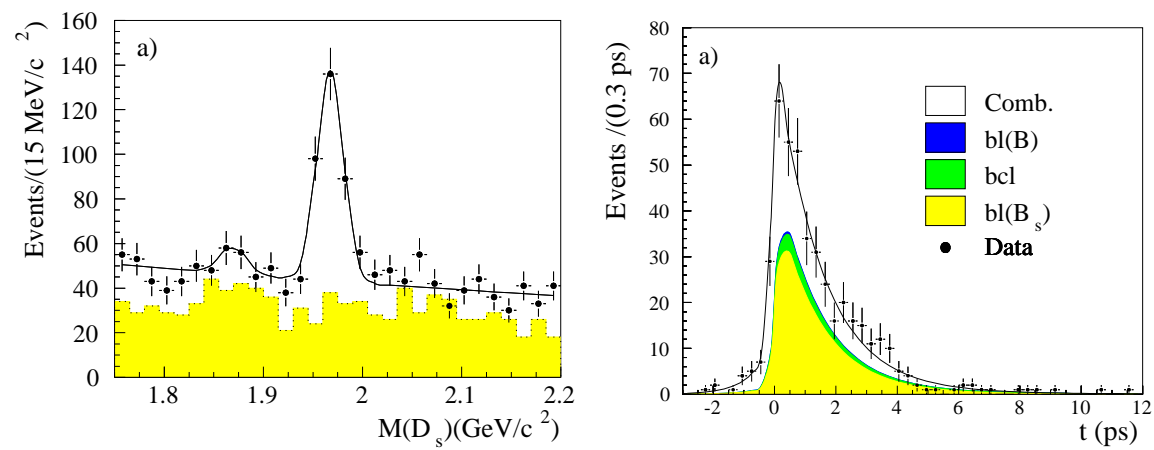

Figure 6: $B_{s}^{0}$ meson lifetime measurement by DELPHI. Left: $D_{s}^{+}$signal associated with $\ell^{-}$. Right: $B_{s}^{0}$ meson proper decay time distribution.

events is observed. Figure 6 shows the combined $D_{s}^{+}$signal (excluding the semileptonic mode) and the $B_{s}^{0}$ decay time distribution. They find $\tau\left(B_{s}^{0}\right)=$ $1.42_{-0.13}^{+0.14} \pm 0.03 \mathrm{ps}$, and $\Delta \Gamma / \Gamma<0.46$. DELPHI also uses $\bar{B}_{s}^{0} \rightarrow D_{s}^{+} h^{-} X$ mode and find $\tau\left(B_{s}^{0}\right)=1.49_{-}^{+0.16}+0.07$ ps and $\Delta \Gamma / \Gamma<0.58$. Other $\Delta \Gamma$ searches include an L3 analysis 23 using inclusive $B$ reconstruction, yielding $\Delta \Gamma / \Gamma<0.67$ and a $\mathrm{CDF}$ analysis 24 using $\sim 600 \ell^{-} D_{s}^{+}$decays, yielding $\tau\left(B_{s}^{0}\right)=1.36 \pm 0.09_{-0.05}^{+0.06}$ ps and $\Delta \Gamma / \Gamma<0.83$. These limits on the width difference, all at $95 \%$ CL, have been obtained by examining decay time distributions and looking for two components.

The $B_{s}^{0}$ lifetime is also measured using modes that are nearly $C P$ eigenstates. ALEPH 25 uses the $B_{s}^{0} / \bar{B}_{s}^{0} \rightarrow D_{s}^{(*)+} D_{s}^{(*)-} \rightarrow \phi \phi X$ signature with $32 \pm 17$ signal events, and finds $\tau\left(B_{s}^{0}\right)=1.42 \pm 0.23 \pm 0.16$ ps. CDF 26 uses $B_{s}^{0} / \bar{B}_{s}^{0} \rightarrow J / \psi \phi$ decays with $58 \pm 12$ signal events, and finds $\tau\left(B_{s}^{0}\right)=$ $1.34_{-0.19}^{+0.23} \pm 0.05$ ps. These decays are not in general pure $C P$ eigenstates. CDF performs a transversity analysis 27 of the $J / \psi \phi$ decays and finds the fraction of the $P$-wave decays $\left(=C P\right.$ odd) to be $\Gamma_{1} / \Gamma=0.229 \pm 0.188 \pm 0.038$. For a similar decay mode $B^{0} \rightarrow J / \psi K^{* 0}$, CDF 27 and CLEO28 find the fraction to be $0.126_{-0.098}^{+0.121} \pm 0.028$ and $0.16 \pm 0.08 \pm 0.04$, respectively.

The above lifetime values may be compared with the world average $B_{s}^{0}$ lifetime measured with flavor eigenstates, $\tau\left(B_{s}^{0}\right)=1.467 \pm 0.058$ ps. This is smaller, albeit large uncertainties, as expected for $C P$-even states. The ALEPH analysis quotes $\Delta \Gamma / \Gamma=0.24 \pm 0.35$ assuming pure $C P$-even composition with $\tau\left(B_{s}^{0}\right)=1.61 \pm 0.10 \mathrm{ps}(\mathrm{PDG} 1996)$.

Since I have reached the page limit, let me conclude here by saying that new data expected in the near future from CLEO-III, Belle, BaBar and Tevatron Run-II should vastly improve the kinds of measurements described here. 


\section{Acknowledgments}

I wish to thank Professor George Hou, my old friend Augustine Chen, and the other members of the local organizing committee for a very nice conference. Also, I would like to thank the experiments for their physics results contributing to this talk. In particular, the following people have provided me with great help in preparing the talk and this manuscript: D. Jaffe, J. Slaughter, K. Baird, D. Jackson, D. Abbaneo, P. Gagnon, R. Hawkings, S. Gentile, S. Blyth and A. B. Wicklund. Professor Joseph Kroll (Penn) has provided brilliant ideas and incredible inspiration as always.

\section{References}

1. N. Isgur and M. Wise, Phys. Rev. Lett. 66, 1130 (1991), J. L. Rosner, Comm. Nucl. Part. Phys. 16, 109 (1986).

2. M. Kobayashi and T. Maskawa, Prog. Theor. Phys. 49, 652 (1973).

3. C. Caso et al., Euro. Phys. J. C 3, 1 (1998).

4. CLEO collaboration, CLEO CONF 99-6 (1999).

5. DELPHI collaboration, Phys. Lett. B 426, 231 (1998).

6. OPAL collaboration, OPAL PN 352 (1998); CLEO collaboration, hep-ex/9901008 (1999).

7. CLEO collaboration, Phys. Rev. Lett. 83, 3390 (1999).

8. CDF collaboration, Phys. Rev. Lett. 81, 2432 (1998); Phys. Rev. D 58, 112004 (1998).

9. L3 collaboration, Phys. Lett. B 465, 323 (1999).

10. OPAL collaboration, OPAL PN 400 (1999).

11. ALEPH collaboration, Phys. Lett. B 425, 215 (1998).

12. CDF collaboration, Fermilab-Pub-99/330-E, submitted to Phys. Rev. D

13. M. Gronau, A. Nippe and J. L. Rosner, Phys. Rev. D 47, 1988 (1993);

M. Gronau and J. L. Rosner, Phys. Rev. D 49, 254 (1994).

14. OPAL collaboration, Phys. Lett. B 327, 411 (1994);

CDF collaboration, Phys. Rev. Lett. 80, 2057 (1998); Phys. Rev. D 59, 032001 (1999).

15. E791 collaboration, Phys. Lett. B 327, 449 (1999).

16. CLEO collaboration, Phys. Rev. Lett. 82, 4586 (1999).

17. I. I. Bigi, These Proceedings (hep-ph/0001003) (2000).

18. SLD collaboration, SLAC-PUB-8206 (1999).

19. ALEPH collaboration, ALEPH 99-005 (1999).

20. LEP $B$ lifetime working group, http://home.cern.ch/claires/lepblife.htm.

21. I. Dunietz, Phys. Rev. D 52, 3048 (1995); R. Fleischer and I. Dunietz, Phys. Lett. B 387, 361 (1996); Phys. Rev. D 55, 259 (1997). 
22. DELPHI collaboration, DELPHI 99-109 CONF 296 (1999).

23. L3 collaboration, Phys. Lett. B 438, 417 (1999).

24. CDF collaboration, Phys. Rev. D 59, 032004 (1999).

25. ALEPH collaboration, ALEPH 98-064 (1998); hep-ex/9905017 (1999).

26. CDF collaboration, Phys. Rev. Lett. 77, 1945 (1996).

27. CDF collaboration, http://www-cdf.fnal.gov/physics/new/bottom/cdf4672/cdf4672.html.

28. CLEO collaboration, Phys. Rev. Lett. 79, 4533 (1997). 